\author{
A. Van der Linden \\ M. Verhoye \\ H. O. Pörtner \\ C. Bock
}

\section{The strengths of in vivo magnetic resonance imaging (MRI) to study environmental adaptational physiology in fish}

Received: 7 July 2004

Revised: 18 October 2004

Accepted: 18 October 2004

Published online: 20 December 2004

(C) ESMRMB 2004

A. Van der Linden $(\bowtie) \cdot M$. Verhoye

Bio-Imaging Lab, Department,

Biomedical Sciences,

University of Antwerp,

Middelheim Campus,

Groenenborgerlaan 171,

2020 Antwerp,

Belgium

E-mail: annemie.vanderlinden@ua.ac.be

Tel.: 003232653230

Fax: 003232653233

H. O. Pörtner · C. Bock

In vivo NMR, Ecophysiology \&

Ecotoxicology, Alfred,

Wegener Institute

for Polar and Marine Research,

27568 Bremerhaven, Germany
Abstract Adaptational physiology studies how animals cope with their environment, even if this

environment is subject to permanent fluctuations such as tidal or seasonal variations. Aquatic organisms are generally more prone to be exposed to osmotic, hypoxic and temperature challenges than terrestrial animals. Some of these challenges are more restraining in an aquatic environment. To date, very few studies have used in vivo magnetic resonance imaging (MRI) to uncover the physiological mechanisms that respond to or compensate for these challenges. This paper provides an overview of what has been accomplished thus far by using MRI to study the environmental physiology of fish. It introduces the reader to the use of small teleost fish such as carp $(12 \mathrm{~cm}, 60 \mathrm{~g})$ and eelpout $(25 \mathrm{~cm}, 50 \mathrm{~g})$ as models for such research and to provide new perceptions into the applicability of MRI tools based on new insights into the nature of MRI contrast. Representative MRI studies have made contributions to the identification of the lack of cell volume repair in stenohaline fish during osmotic stress. They have studied the underlying physiological mechanisms of brain anoxia tolerance in fish and have qualified the role of the cardio-circulatory system in setting thermal tolerance windows of fish.

Keywords MRI · Fish · Adaptational physiology

Abbreviations ADC: Apparent diffusion coefficient - ATP:

Adenosine triphosphate - BOLD: Blood oxygenation level dependent (contrast) - BPG:

Brain-pituitary-gonadal (axis) - CBF: Cerebral blood flow $\cdot$ CBV: Cerebral blood volume - DW: Diffusion weighted - FOV: Field of view - GE: Gradient echo - HPA:

Hypothalamic-pituitary-adrenal (axis) $\cdot \mathrm{MO}_{2}$ : oxygen consumption - MRI: Magnetic resonance imaging - MRS: Magnetic resonance spectroscopy $\cdot$ NMR: Nuclear Magnetic resonance $\cdot$ RF: Radio frequency · RVI: Regulatory volume increase - SE: Spin echo - STW: Standard water $\cdot \mathrm{T}_{2}$ : Transverse relaxation MRI parameter $\cdot \mathrm{T}_{2} \mathrm{~W}$ SI: $\mathrm{T}_{2}$-weighted signal intensity $\cdot \mathrm{T}_{2} *$ : $\mathrm{T}_{2}$ star $\cdot \mathrm{TE}$ : Echo time $\cdot \mathrm{T}_{\mathrm{c}}$ : Critical temperature - TOF: Time of flight $\cdot \mathrm{T}_{\mathrm{p}}$ : Pejus temperature $\cdot \mathrm{TR}$ : Repetition time 


\section{Introduction}

Animal life is found in a wide range of environmental conditions that range from polar oceans, frozen lakes, flowing rivers to cold and hot deserts with minimal availability of water. Comparative and environmental physiology addresses how animals cope with the problems posed and exploit the opportunities offered by their particular habitat [1]. Study of organismal functions under environmental extremes and of the exceptional physiological solutions found is relevant not only from the point of view of the animal enthusiast, who wants to understand how animals work; the comparative approach also asks how these functions were shaped during evolution and how they are limited in capacity and with respect to environmental influences. How did the "norm" of physiological functions evolve to support the generalist in normal conditions? How were "normal" functions modified to solve particular challenges in environmental extremes? Usually such special solutions go hand in hand with a specialization on specific habitats. Such specialization implies limited adaptational capacity once environmental conditions change. They therefore play a key role in understanding the effects of anthropogenic influences on ecosystems. For example, the physiological mechanisms setting thermal tolerance and defining thermal sensitivity have recently come into focus due to rising interest in the effects of climate change on organisms and ecosystems. Such information will not only support an understanding of how temperature and its oscillations shape biogeography and species survival $[2$, 3] but is also relevant to understand how climate variability throughout earth history has influenced the directions of animal evolution [4].

Last but not least, comparative and environmental physiology also leads to an understanding of the phylogenetic background of physiological functions found in the human body. It allows to put into perspective the functional limits and constraints of these functions, which may become important under pathological conditions. Solutions and mechanisms of stress and survival found in animals in an environmental context may even support a mechanistic understanding of normal and pathological function in a medical context.

Study of environmental adaptation has always depended on the availability of suitable animal models as well as of a suitable spectrum of methods. Recent insight emphasizes that it is not possible to comprehend fully the scope and limits of adaptation, based exclusively on molecular and biochemical techniques (e.g. [5]) or exclusively on ecological approaches. Driving forces for adaptation operate at ecosystem levels, whereas the scopes and limits of adaptation originate from an integration of molecular functions into cellular, tissue and whole-organism functioning (e.g. $[3,4]$ ). An integrative approach is inherent to environmental physiology, which bridges the various fields and relies on the application of techniques that allow integration of the various levels of molecular to organismal functioning.

Such a spectrum of techniques is offered by nuclear magnetic resonance spectroscopy and imaging tools (MRS and MRI) which have more recently become available for the study of environmental adaptation. These techniques are suitable for the combination of invasive studies at molecular to organismic levels with the capability to monitor physiological functioning non-invasively in the intact organism. More recently, MRI techniques were modified such that suitable animal models, for example from aquatic habitats, have been studied online under simulated environmental conditions ([6-10]: fish; [11]: molluscs; [12-14]: crustaceans).

The present review provides an overview of what has been accomplished thus far using MRI to study the environmental physiology of teleost fish (bone fish, as opposed to cartilage fish such as sharks). It aims to introduce the reader to the use of teleost fish as animal models to understand the underlying mechanisms of general physiological processes and to validate imaging techniques by providing new insights into the nature of the MRI contrast.

\section{Special precautions when performing in vivo MRI on fish}

\section{An RF antenna mounted in an aquatic environment}

As a consequence of the MRI method being particularly sensitive to the detection of water and water motion, studying aquatic organisms requires several precautions. Fish need a continuous flow of aerated water to irrigate their gills during the MRI measurements. This requires first of all a water-tight animal bed in the shape of a pipe ensuring that no water comes into contact with the gradient insert. Even more stringent is the development of a customized animal holding system with the required RF antenna mounted in such a way that it does not come into contact with the water and exclusively covers the regions under study, excluding irrigated gill areas or other areas exposed to water flow, to avoid artefacts in the image due to water motion. With the gills being in the immediate vicinity of the brain, this problem becomes worse when performing brain studies. One solution is to use a Helmholtz (transmit/receive) radio frequency (RF) antenna mounted like a headphone covering the upper part of the head of the fish, including the brain but excluding the irrigated gill area. The RF antenna is mounted in a water-free compartment included in the fish restraining chamber (Fig. 1). Alternatively this antenna can be combined with - or be replaced by - a circular surface RF antenna mounted on top of the fish head.

When investigating body parts other than the head, and in an attempt to avoid undesired flow artefacts from 

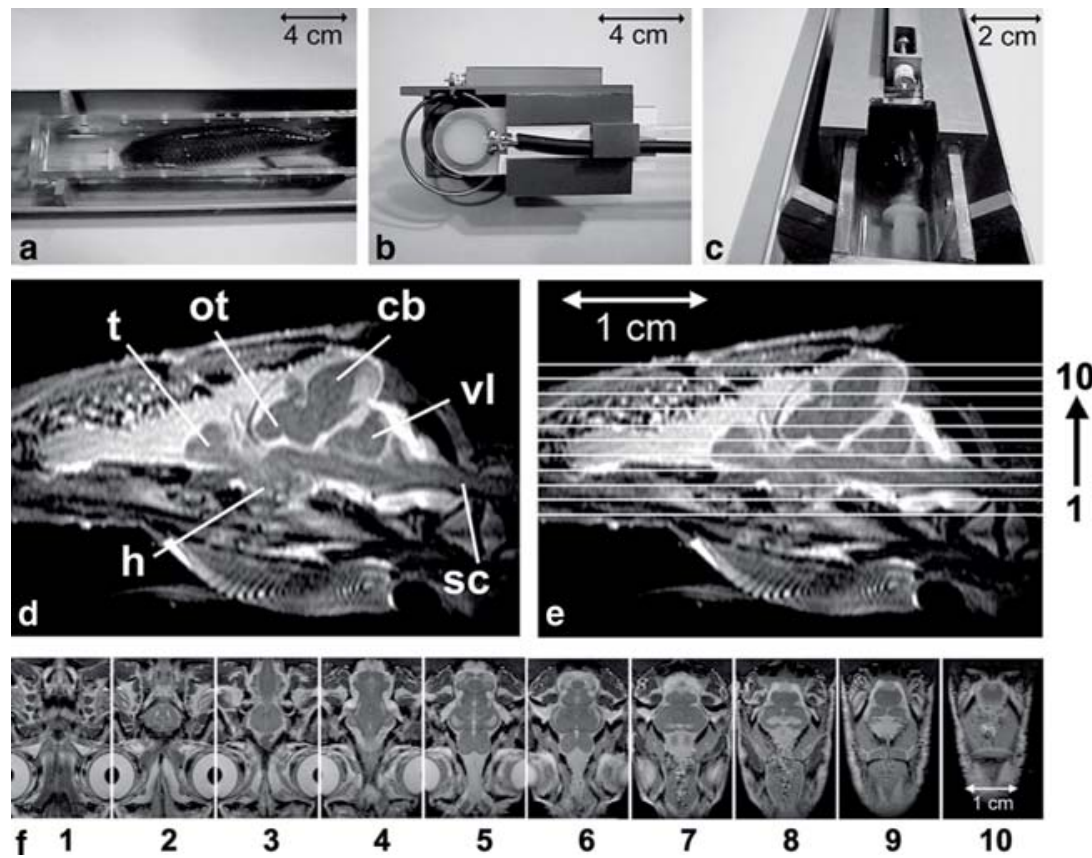

\section{0}

Fig. 1 a Animal holding device containing an anaesthetized carp. b Dedicated RF headphone antenna. c Animal holding device with RF antenna mounted so as to allow MRI of the head of the fish while not being in contact with the flowing water. A constant irrigation of the gills is ensured as outlined in the text. This setup is inserted in the bore of the magnet of the MR instrument. $\mathbf{d}-\mathbf{f}$ provide high-resolution MRIs obtained on anaesthetized common carp with the setup shown in (c). With a spatial resolution of 156 micron, $\mathbf{d}$ and e provide a midsagittal image through the head of the fish showing the brain and the spinal cord. The hyperintense tissue surrounding the brain is the meninx, a jelly-like mass, filling the cranial cavity. e and d are identical images but e illustrates, superimposed, the horizontal MRI sectioning plane with consecutive slices of 1-mm thickness (From 1 to 10) shown in (f). (f1-10) represent an entire set of consecutive horizontal MRIs obtained from bottom to top of the carp brain showing the following structures: (h) hypothalamus, (ot) optic tectum, (vl) vagal lobe, (sc) spinal cord, (t) telencephalon, (cb) cerebellum. Similar horizontal MRI slices were obtained with $\mathrm{T}_{2}$ - and diffusion-weighted settings and provided information on the water balance in different brain regions (adapted from [8])

the surrounding water, the anaesthetized fish can be immobilized according to Van den Thillart et al. [15]. An airfilled bag is used to cover the measured body region, thereby avoiding water flow in the region covered by the RF coil (Fig. 2). The fish is inserted into a restraining tube with head and tail regions of the fish freely exposed to the environmental water and with a tube for water inflow in its mouth. Figure 3 illustrates the MR imaged region using this setup, which is the abdomen of the fish.

Studying marine fish: salt water as an extra challenge for MRI

Using seawater has immense implications for the use of both MR hardware and sequences. The salinity of sea

water decreases the quality factor $(Q)$ of nuclear magnetic resonance (NMR) coils [6,16]. Tuning and matching capacitors must be readjusted to minimize signal reflection and frequency shifts. Sea water conductivity may induce reflections of more than $50 \%$ and frequency shifts of up to $3 \mathrm{MHz}$ due to dielectric losses, depending on the resonance frequency and the amount of sea water inside the probe [10]. Consequently, RF-pulse length and power both decrease with increasing salinity (up to $11 \mathrm{~dB}$ ) [10]. Specially adapted NMR probes for high conductivity samples and RF power (up to $2 \mathrm{~kW}$ ) can reduce these negative effects of salt water. The combination of both a cylindrical resonator for a homogeneous excitation profile and a detection surface coil for improved spatial resolution operating in crossed coil mode enhances the signal-to-noise ratio by further reducing dielectric losses. Additionally, excitation pulse shapes, gradients and pulse sequences can be customized. For instance, gradient echo sequences using low flip angles will reduce the required RF power significantly in comparison to conventional spin echo MRI.

The impact of salinity on MRI acquisitions is also illustrated in the study of Van der Linden et al. [7] since twice the number of acquisition averages were acquired for fish exposed to sea water as compared to the control fish that remained in fresh water.

Anaesthetized, conscious restrained and even unrestrained fish

Fish can be anaesthetized by adding $0.011 \%$ MS 222 (ethyl meta aminobenzoate metanesulfonic acid salt 98\% Sigma) to $\mathrm{pH}$-controlled water. The main reason for anaesthesia 

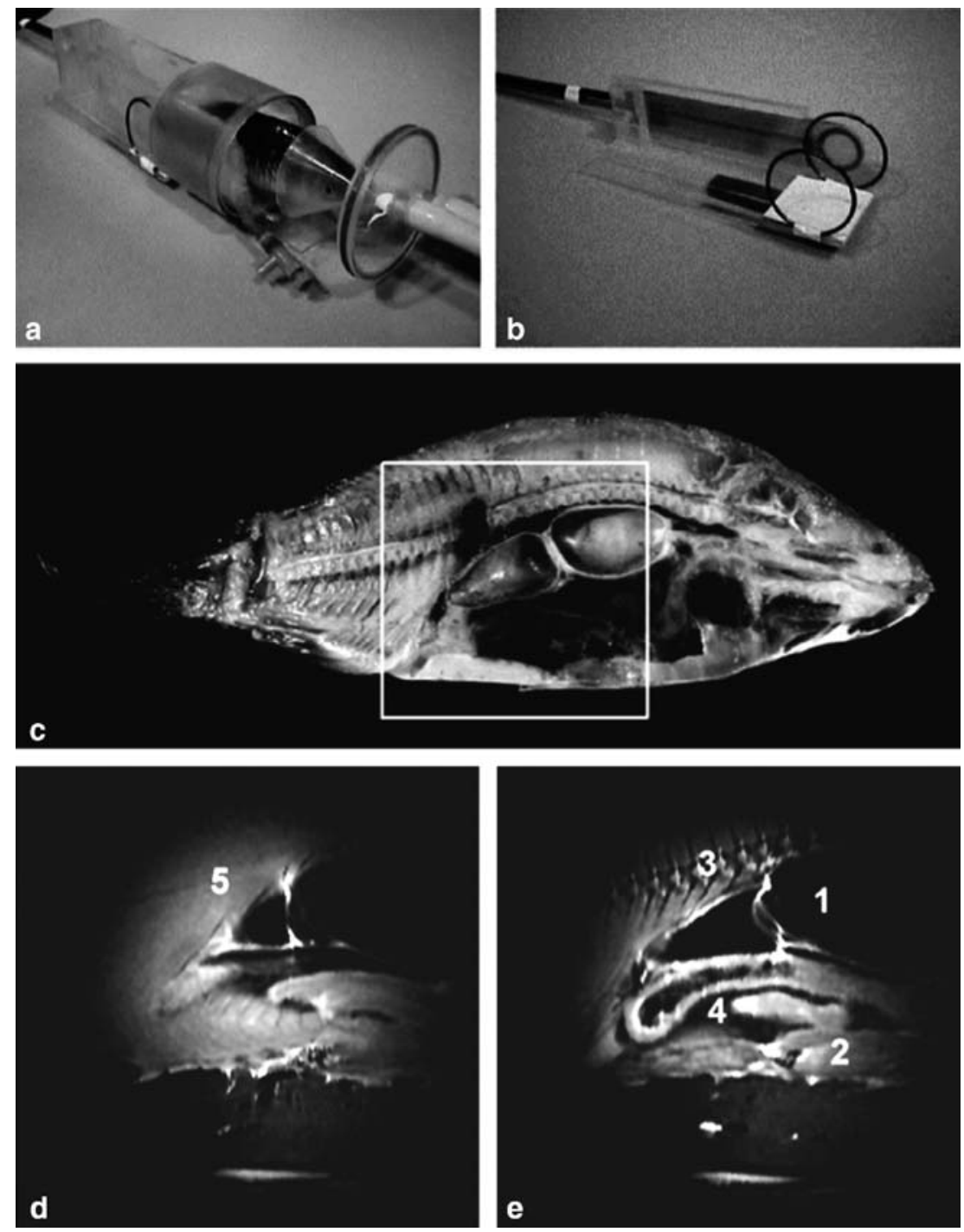

Fig. 2 a Animal chamber and water flow-through system developed to image the belly of restrained fish. Once the anaesthetized fish is positioned between the airbags, the flow-through system can be closed and the RF antenna (b) positioned such that it covers the belly region of the fish. From now on the fish receives fresh water without anaesthetics through a tube fixed in the mouth and remains unanaesthetized but restrained in the closed flow-through system during the entire MRI experiment. Environmental temperature, oxygen, $\mathrm{pH}$ and salinity of the water can be varied and controlled as outlined in the text. c Macroscopic image of a frozen carp, cut midsagittally. The insert shows the area covered by the RF antenna and $\mathbf{d}$ and $\mathbf{e}$ represent the corresponding $\mathrm{T}_{2}$-weighted MRI obtained in vivo, revealing the following structures: (1) the swim bladder; (2) the intestine; (3) the vertebral column; (4) the liver; and (5) the muscle (adapted from [7])

is to immobilize the animals or anaesthetize them if the experiment is considered too stressful. To evaluate the impact of anaesthesia during an anoxic stress, non-anaesthetized but curarized fish were investigated. The MRI

outcome was similar, but the fish did not recover, which prevented repeated measurements of the same specimen. This finding was in contrast to the effect of MS 222 which was applied in such a low dose that the animals could easily be kept anaesthetized for up to $6 \mathrm{~h}$, the duration of an entire experiment, and still recover uneventfully from the experimental treatment [8].

Subsequent to anaesthesia induction, the fish can be mounted in an experimental system, restrained if needed as outlined above - and inserted in the bore of the magnet of the MR instrument. Aerated water with anaesthetics can then be pumped $(500 \mathrm{ml} / \mathrm{min})$ from an aquarium to a tube fixed in the mouth of the fish. If the fish is properly immobilized by an air-filled bag - as outlined above - it can remain immobilized even when switched to water without anaesthetics.

If bottom-living, rather inactive fish are used [9], anaesthesia and restraining can be successfully avoided and analyses carried out under more natural conditions. 


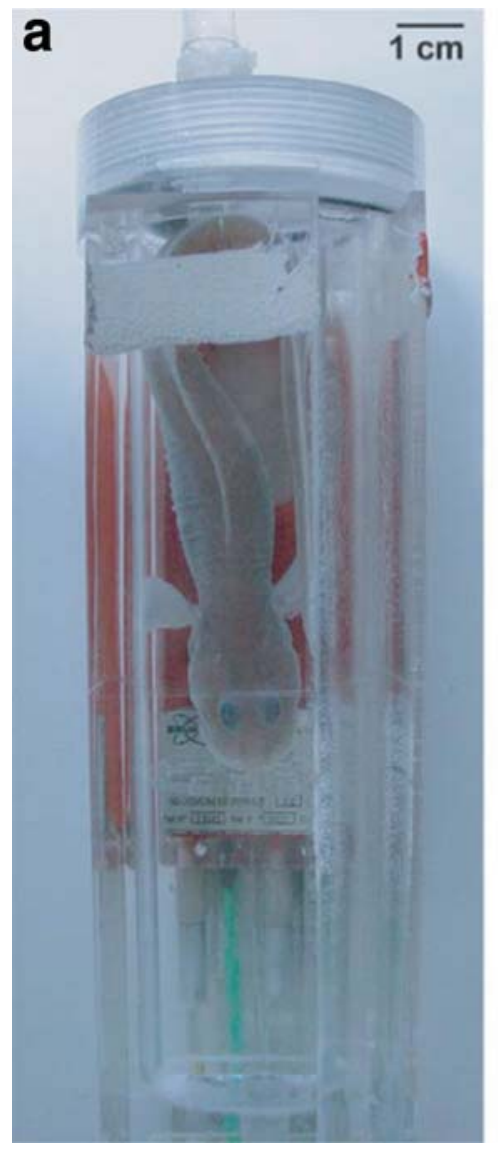

Fig. 3 a Flow-through chamber for in vivo MRI measurements of non-anaesthetized and unrestrained fish (max. animal length $40 \mathrm{~cm}$ ), containing a specimen of the Antarctic eelpout Pachycara brachycephalum. Variable slide barriers were used to fit the size of the fish to the chamber and the position of the surface coil (in red). The posture of the animal is typical for eelpout, which tend to hide in narrow tubes. b MRI of the eelpout under control conditions $\left(\mathrm{T}=0^{\circ} \mathrm{C}\right)$ : the middle image is an anatomical coronal MRI of the fish positioned as illustrated in (a). On the left, the axial flow-weighted MRI shows: (1) the aorta dorsalis and (2) vena cava posterior as bright spots, and additionally (3) the stomach, (4) the dorsal muscle, (5) the spine and (6) the tail. On the right, the $\mathrm{T}_{2}^{*}$-weighted MRI displaying the tissue oxygenation shows: (1) the dorsal white muscle, (2) the spine, (3) the blood vessels, (4) the stomach, (5) the liver and (6) the tail (adapted from [9])

Although these fish had the opportunity, they did not attempt to move during MRI acquisitions. Two variable slide bars at both ends of the animal chamber secured an optimal position of the animal relative to the RF surface coil. The unrestrained fish was placed into the chamber without the need for anaesthesia during experimental periods of more than a week (Fig. 3). Animals showed only mild signs of stress due to handling, indicated by a transient reduction of phosphocreatine or increase of inorganic phosphate levels in white muscle as derived from in vivo ${ }^{31} \mathrm{P}$-NMR spectroscopy $[9,17,18]$. Moreover, most sessile fish species, including those from polar areas, have a very low metabolic rate. The fact that non-feeding for periods of up to several weeks does not push these fish into starvation makes them excellent animals for a long-term stay in the magnet.

\section{Benefits of performing in vivo MRI on teleost fish}

\section{MRI sequences, contrast and signal-to-noise ratio}

Blackband and Stoskov [6] demonstrated applicability of MR imaging and localized spectroscopy to aquatic as well as marine organisms with classic MRI and MRS methods. Within a suitable aquatic life-support system conventional spin echo and inversion recovery sequences as well as the STEAM technique for localized spectroscopy yielded high-resolution data [6]. Technological possibilities have advanced since then such that, recently, even fast gradient echo (FLASH and Snapshot Flash) MRI and fast spin echo MRI (RARE) were applied to marine teleost fish [10]. Unwanted signals from surrounding water were excluded by taking into account the flow direction of the surrounding water relative to the phase gradient. Consequently, no residual water was visible in RARE images 
and tissue contrast was increased [10]. Moreover, FLASH and RARE sequences are very sensitive to susceptibility changes and good field homogeneity is most important for $\mathrm{T}_{2}^{*}$-weighted MRI or blood oxygenation level dependent (BOLD) imaging. In contrast to classic MRI studies on rodents, fish without swim bladders located in a cylindrical animal chamber filled completely with water can easily be used for $\mathrm{T}_{2}^{*}$-weighted MRI studies, using automated shimming $[9,13]$. This setup supported localized ${ }^{1} \mathrm{H}$ NMR spectroscopy using a PRESS sequence with typical line widths of around $10 \mathrm{~Hz}$ and revealed, for instance, different spectra between adult and embryos in viviparous fish (eggs hatch in the mother which results in 'giving birth' instead of "laying eggs") [10]. Since aquatic organisms and most fish have low metabolic rates (see above) cardiac imaging without ECG triggering is possible with standard MRI sequences $[6,13]$. Taking the special prerequisites of the surrounding water into account, any MR imaging and spectroscopy application can be applied to teleost fish in the same way as in mammalian animal models.

Physiological monitoring in fish: the similarity with a perfused organ

By positioning the fish in the bore of the magnet whilst receiving water through a fixed mouth tube from a reservoir outside the magnet, the animal is comparable to a "perfused organ". Since the fish gills represent $70 \%$ of the organism's exchange surface with the environment, and since gill epithelia are responsible for gas exchange, changing gas partial pressures in the water immediately change these parameters in the blood. Moreover, fish are ectotherms, with body temperatures close to those of the environment. Changing water temperature will immediately change the temperature of blood and, as a consequence, of the entire body. This "perfused fish" setup allows monitoring of temperature and gas tension of the environmental water to substitute for monitoring body temperature and (as a first approximation) for monitoring blood gasses. Water oxygen concentration and temperature are recorded online with an OXY 325-B probe, and $\mathrm{pH}$ with a PHM 93 probe (Radiometer Copenhagen; WTW). The probes are connected to a PC with Lab Windows software and included in the water circuit as close as possible to the mouth inlet to enable rapid registration of experimentally induced changes. The probes' position is just outside the bore of the magnet $(40 \mathrm{~cm}$ away from the fish mouth) since it remained unaffected by the magnetic field strength. For temperature incubation experiments, fluoroptic temperature sensors (Luxtron 504, Polytechnic, Germany) were installed directly in the animal chamber and in the reservoir tank. Temperature was recorded continuously using a PowerLab system and stored onto a PC with Chart software (ADInstruments, Germany).
Teleost fish as models to study response to environmental change

As outlined in the introduction, the objective of adaptational physiology is to study how animals cope with the environment they live in, even if this environment changes permanently as during tidal or seasonal variations. Aquatic organisms are, in some cases, exposed to larger environmental changes than terrestrial animals. Indeed, except for desert animals, terrestrial animals are less frequently exposed to osmotic challenges and to a lesser extent to hypoxia (since air is a much richer source of oxygen). Furthermore, although temperature fluctuations are smaller, water breathers may face more demanding challenges since oxygen content is lower and warming impairs both water oxygen availability and utilization. Oxygen limitations were recently discussed to represent the first line of thermal limitation as a unifying principle in animals [2], with special features in fish [19].

Since fish are ectotherms and display lower metabolic rates and higher resistance to conditions like hypoxia or anoxia this allows for a broader range of environmental changes to be sustained and assessed on longer time scales than in euthermic mammals. Moreover, changing environmental conditions such as between normoxia and hyperoxia, anoxia or hypoxia can easily be created by equilibrating the water in the reservoir tank with the appropriate gas mixture using a gas-mixing pump (Wösthoff, Germany). Temperature changes can be accomplished by means of cryostats or heaters in the reservoir tank. To establish that the fish experiences, while in the magnet, rapid transitions from anoxia to normoxia, cold to warm, or salt to fresh water and vice versa, it suffices to set up two aquaria with different experimental conditions and a three-way valve close to the fish mouth, allowing a rapid switch between the two.

\section{Physiological MRI studies in fish: examples}

Environmental changes in an aquatic environment, like those in temperature, gas tensions or osmolarity, can be acute or chronical, for example in intertidal zones, where fish are swimming up or downstream, or during seasonal or diurnal fluctuations. Examples outlined below will illustrate how in vivo MRI can be an excellent tool to provide insights into how changing environmental parameters affects the physiology of fish.

\section{Fish brain response to acute anoxia}

While the mammalian brain is irreversibly damaged after a few minutes of anoxia, some vertebrates such as the crucian carp (Carassius carassius), a close relative of the goldfish, survive for 1 or 2 days under anoxia at 
room temperature. These fish are able to maintain brain adenosine triphosphate (ATP) levels during anoxia by combining an increased rate of anaerobic (glycolytic) ATP production with a depressed rate of energy use [20]. Its anoxia-intolerant relative, the common carp (Cyprinus carpio), can only survive for $2 \mathrm{~h}$ under anoxia at room temperature during which time a continuous fall in brain ATP levels is observed [21,22]. As a consequence, brain swelling and edema as well as irreversible brain damage may occur in this species.

For the respective analyses, both fish species $(n=$ 5) were submitted to $2 \mathrm{~h}$ of anoxia and subsequent normoxic recovery while monitoring cell swelling events with $\mathrm{T}_{2-}$ and diffusion-weighted MRI as usually carried out in clinical and experimental stroke research (e.g. [23-27]. While in the magnet, anaesthetized and restrained fish were submitted to a period of normoxia (the water was bubbled with air to maintain $100 \%$ oxygen saturation $=9 \mathrm{mg} \mathrm{O}_{2} / \mathrm{ml}$ ), $2 \mathrm{~h}$ of anoxia (the water was bubbled with nitrogen) and allowed to recover under normoxia for $100 \mathrm{~min}$. MR Imaging was applied to fish with the head mounted in a custom-made RF headphone antenna of 40-mm diameter (Fig. 2). The entire setup was inserted in the bore of a horizontal 7-T magnet of an SMIS MRI system (UK). Consecutively, T2-weighted gradient echo (GE) images $(\mathrm{TE} / \mathrm{TR}=12 \mathrm{~ms} / 500 \mathrm{~ms}$, acquisition matrix $128 \times 128$ ), different $\mathrm{T}_{2}$-weighted spin echo $(\mathrm{SE})$ images $(\mathrm{TE} / \mathrm{TR}=18 / 2000,34 / 2000,50 / 2000)$ and different diffusion-weighted (DW) spin echo images (TE/TR $=50 / 2000$, diffusion-sensitizing gradient pulse in the $\mathrm{X}$ direction, duration $(\delta)$ of $10 \mathrm{~ms}$, diffusion-gradient separation time $(\Delta)$ of $15 \mathrm{~ms}$, diffusion gradient ramp time of $1 \mathrm{~ms}, b$ values of $b_{0 x}=0, b_{1 x}=2.993, b_{2 x}=6.736 \times 10^{8} \mathrm{~s} / \mathrm{m}^{2}$, acquisition matrix $256 \times 128)$ were acquired at the level of the brain of the fish. The field of view (FOV) was $40 \mathrm{~mm}$ and the spectral width was $25 \mathrm{kHz}$. Two averages were taken and the 12 acquired consecutive $1-\mathrm{mm}$ slices covered the entire fish brain. The GE $\mathrm{T}_{2}{ }^{*}$-weighted images, which showed visible darkening when the blood perfusing the brain became anoxic (increased amount of paramagnetic deoxy$\mathrm{Hb}$ ), served to control appropriate vascular circulation, heart function and gill perfusion of the fish during the experiment. For each time point, the $\mathrm{T}_{2}$ and apparent diffusion coefficient (ADC) values were calculated on a pixel-by-pixel basis.

The study clearly showed that, although both common carp and crucian carp survived $2 \mathrm{~h}$ of anoxia at $18^{\circ} \mathrm{C}$, the response of their brains to anoxia was quite different. As expected, the anoxic crucian carp showed no signs of brain swelling or changes in brain water homeostasis. In contrast, the anoxic common carp brain suffered from serious cell swelling as indicated by the ADC values, which showed a drop by $53 \pm 8 \%$ after $2 \mathrm{~h}$ of anoxia. Upon normoxic recovery some brain areas regained their control ADC values while others did not. A net water

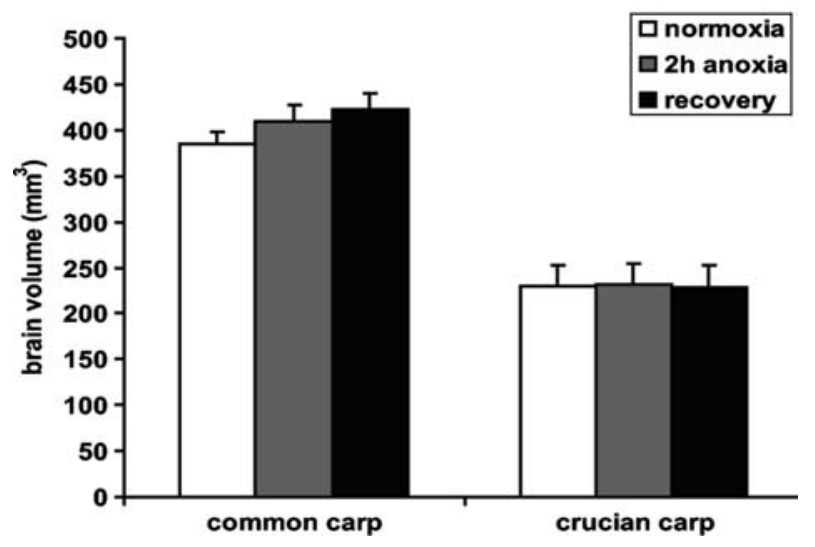

Fig. 4 Mean brain volume $\left(\mathrm{mm}^{3}\right)( \pm$ SD) of common carp $(n=5)$ and crucian carp $(n=5)$ when exposed consecutively to normoxia, $2 \mathrm{~h}$ of anoxia and $100 \mathrm{~min}$ of normoxic recovery. The data also reveal that, although the common and crucian carp were equally sized, the common carp has a much larger brain (adapted from [8])

gain was discerned from the average $T_{2}$ values, which were $58 \pm 10 \mathrm{~ms}$ during the initial normoxic conditions but increased significantly to $67 \pm 7 \mathrm{~ms}$ after $100 \mathrm{~min}$ of normoxic recovery. This presumably reflected the observed brain volume increase $(6.5 \%)$, which continued (by $10 \%$ ) during 100 min of normoxic recovery (Fig. 4). Like in many fish, the brains of both studied carp species are embedded in a soft mass of jelly-like tissue called meninx, and the cranial space surrounding the brain is much larger than the brain volume. Common carp were able to recover from this insult, indicating that these changes were reversible. It can be suggested that the oversized brain cavity and the jellylike meninx, seen in many ectothermic vertebrates, allows brain swelling during energy deficiency without a resultant increase in intracranial pressure and global ischemia, as observed in mammals under similar circumstances.

Responses to acute and prolonged salt exposure in freshwater fish

Regulation of internal water balance and ion concentrations are critical in fish since they either live in the hyper-osmotic marine environment or in hypo-osmotic fresh water. Depending on the level of tolerance displayed to changes in external salt concentration, two types of freshwater fish can be distinguished: stenohaline (narrow range of tolerance) and euryhaline fish (broad range of tolerance), which display different physiological responses when exposed to salt water. Stenohaline freshwater fish will hyper-regulate their internal osmolarity to allow the continuous influx of water necessary for the elimination of waste products via the kidneys. Euryhaline freshwater teleosts exposed to salt water are hypo-regulators that prevent net water loss by drinking salt water and keep the composition of the body fluids constant by salt excretion 
[28]. Generally, when animals or cells are exposed to a hypertonic external solution, the cell volume decreases by water loss and must be regulated during the process of regulatory volume increase (RVI), which is accompanied and caused by shifts of osmolytes [28-30].

The common carp (Cyprinus carpio) is a well-known example of a stenohaline freshwater fish [31]. When exposed to $1 \% \mathrm{NaCl}$ it survives osmotic stress for more than a month but eventually the fish die revealing that no acclimation took place. Lack of regulatory volume increase might be the reason for enhanced mortality. To that end, we introduced in vivo MRI to investigate the dynamic changes in internal water balance, particularly in liver and muscle of carp, upon prolonged exposure to $1 \%(10 \mathrm{~g} / \mathrm{l})$ $\mathrm{NaCl}$ and correlated these data with plasma osmolarity data obtained from the same specimens [7].

Two weeks before starting the experiments, carp were transferred to moderately hard standard water (STW) according to standard methods (American Public Health Association, 1989) and kept at room temperature (22 \pm $2^{\circ} \mathrm{C}$ ). Carp were fed daily ad libitum with pond sticks (Tetrapond, Henkel) and excess food was removed 15 min after feeding. Salt exposure was accomplished by adding $10 \mathrm{~g}$ of $\mathrm{NaCl}$ to $1 \times l$ STW $(1$ weight $\%=1 \mathrm{wt} . \%)$. Thirty-six fish were transferred from the aquarium filled with STW to the tank with the $1 \mathrm{wt} . \% \mathrm{NaCl}$. Carp were again fed daily ad libitum but due to loss of appetite the quantity of food given was reduced by $50 \%$.

After 1, 2, 4, 7, 14 and 21 days of salt exposure, carp ( $n=6$ for each time point) were submitted to an in vivo MRI protocol. They were positioned and restrained in an animal chamber and MRI was applied without anaesthesia as outlined above to avoid the influence of anaesthesia on osmoregulatory capacity. Results were compared with those obtained for fish kept in STW $(n=$ 12). After MRI measurements, blood samples were taken to determine plasma osmolarity and ion concentration. Sagittal diffusion-weighted MRI was performed on the same 7-T SMIS NMR instrument (Guildford, UK) mentioned in the previous chapter and using the same RF antenna, but turned upside down to image the abdomen (Fig. 2). The same diffusion-weighted spin echo sequence was applied except for the diffusion gradient direction $(Y)$. Field of view $(F O V)=50 \mathrm{~mm}$ with an in-plane resolution of $(195 \times 195) \mu \mathrm{m}^{2}, 16$ slices with slice thickness of $1 \mathrm{~mm}$, TE/TR $=36 / 2000 \mathrm{~ms}$, number of averages: $n=2$ for STW, and $n=4$ for salt-treated fish. The $b_{0 y}$ image was used as a $\mathrm{T}_{2}$-weighted image. An Eppendorf tube filled with water, used as an internal reference for SI, was positioned within the imaged region to ensure standardization of the measurements, and $\mathrm{T}_{2}$ signal intensity $\left(\mathrm{T}_{2 \mathrm{w}} \mathrm{SI}\right)$ values of the muscle and liver tissues were expressed relative to the SI of the water reference.

It seemed that the osmolarity of a $1 \% \mathrm{NaCl}$ solution (about $324 \mathrm{mOsm} / \mathrm{kg}$ ) equalled the initial plasma osmolarity of the fish when kept in fresh water $(328 \pm 19 \mathrm{mOsm} / \mathrm{kg})$

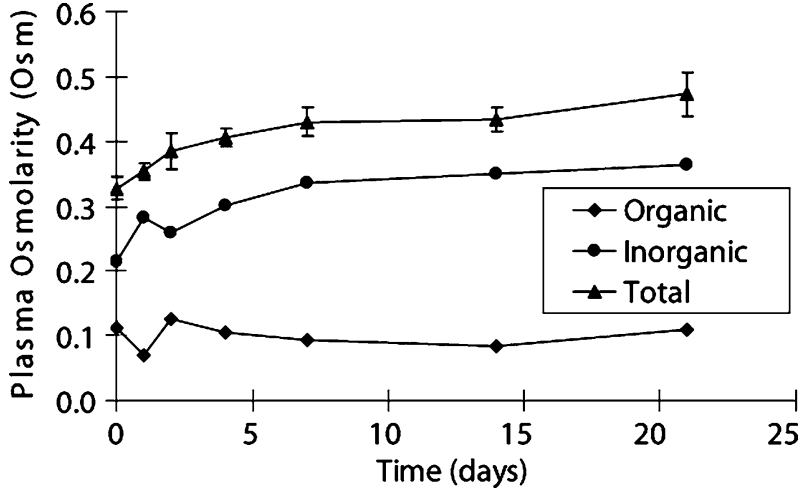

Fig. 5 Plasma osmolarity and the contribution of plasma inorganic and organic compounds to plasma osmolarity of the common carp when exposed for up to 21 days to $1 \% \mathrm{NaCl}(n=6$ for each time point) (adapted from [7])

(Fig. 5). The obtained plasma osmolarity data confirmed that carp is a hyper-regulator, since upon exposure to $1 \% \mathrm{NaCl}$, the internal osmolarity (plasma) of the carp attained values which were 30 to $45 \%$ higher than the external value, supporting passive influx of water. Concomitant with but unlike the plasma data, data on water balance were obtained in an in vivo non-invasive manner and on conscious fish. These data demonstrated that upon exposure to $1 \% \mathrm{NaCl}$, liver and muscle of the stenohaline common carp showed signs of dehydration during the first days of exposure (decreased $\mathrm{T}_{2}$ values, shown in Fig. 6a). Upon osmotic adjustments of the plasma, which reached a plateau after 7 days (Fig. 5), rehydration of the investigated tissues occurred (Fig. 6a). However, no cell volume repair occurred but continuous increase of the extracellular volume was observed instead (increased ADC values with increased $\mathrm{T}_{2}$, shown in Fig. 6a, b). Liver and muscle tissue displayed the same type of response to the osmotic challenge but to a different degree. Even within 21 days, extracellular volume did not attain equilibrium values but continued to increase (Fig. 6b). By that time, the liver had gained twice the control $\mathrm{T}_{2}$ SI (Fig. 6a). All of this explains why carp do not survive exposure to $1 \% \mathrm{NaCl}$ for longer than 1 or 2 months.

\section{Thermal tolerance of marine fish}

With respect to temperature tolerance, polar ectotherms are stenothermal (e.g. Antarctic fish species living at constant temperatures around $0^{\circ} \mathrm{C}$ ) and display low tolerance to temperature changes, whereas eurythermal animals (e.g. temperate fish confronted with seasonal fluctuations in temperature) have a much broader thermal tolerance window. The eelpout fish family ranges from temperate zones and the deep sea to polar regions, harbouring both stenothermal and eurythermal family members and therefore provides suitable animal models for 

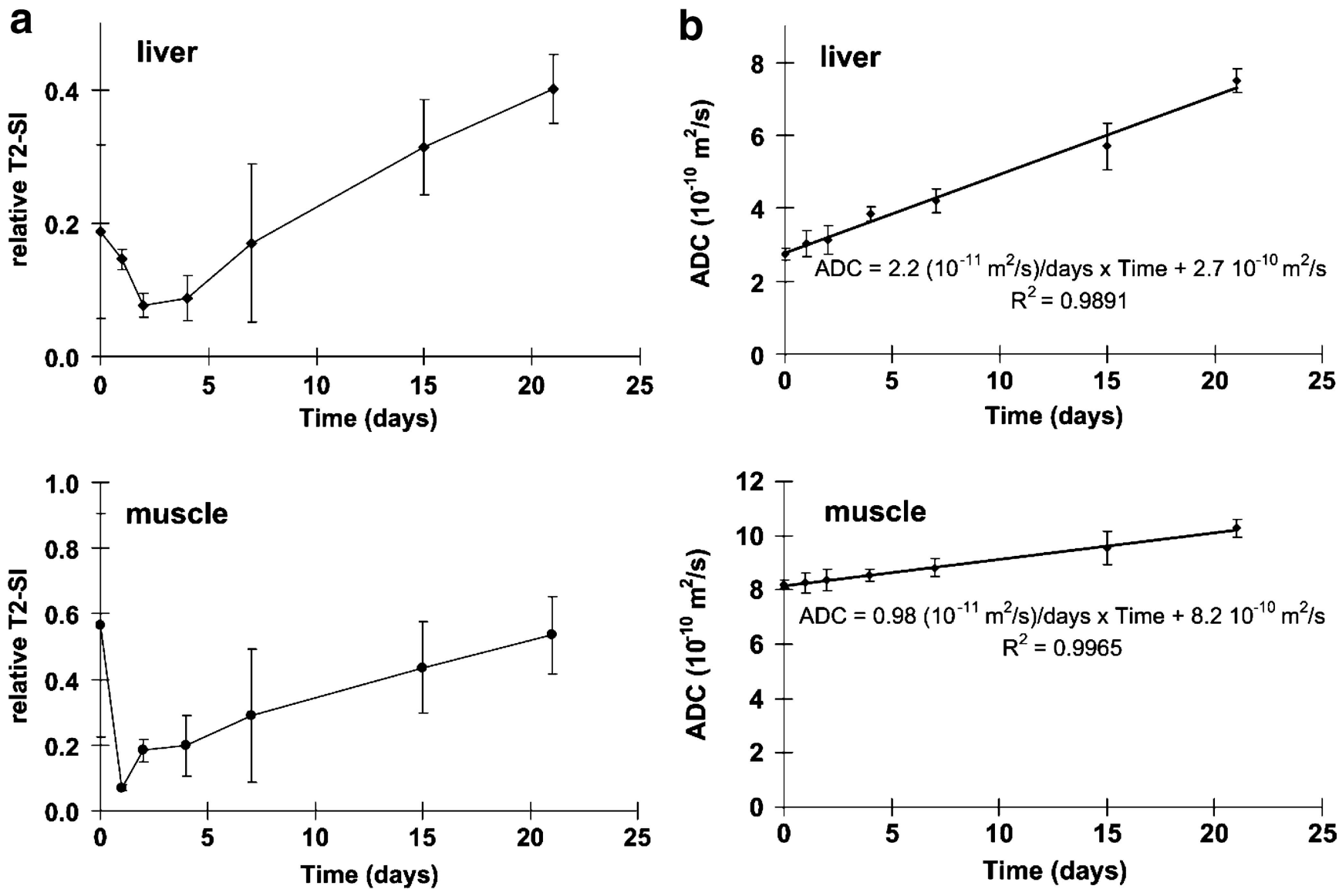

Fig. 6 a Relative (to a water phantom) T-2-weighted signal intensity $( \pm \mathrm{SD})$ (providing information on the tissue water content) and $\mathbf{b}$ ADC values $( \pm \mathrm{SD})$ ( providing information on the ratio of intra-versus extracellular volume), determined in liver (top) and muscle (bottom) of the common carp upon exposure to $1 \% \mathrm{NaCl}(n=6$ for each time point) (adapted from [7])

comparative physiological studies of temperature acclimation and adaptation $[17,18]$.

The physiological mechanisms behind thermal tolerance limits are currently under investigation, with respect to the question of how animals maintain function at different temperatures and how they are able to adjust their thermal tolerance windows $[2,32]$. A recent concept based on data collected in various groups of marine ectotherms is the hypothesis that thermal tolerance in animals is limited by oxygen availability to tissues [2]. Briefly, limits of thermal tolerance during warming and cooling are indicated by threshold temperatures (pejus temperatures, $T \mathrm{p}$, and critical temperatures, $T \mathrm{c}$ ), where oxygen supply decreases beyond pejus temperatures, resulting in a drop of aerobic scope. Full availability of aerobic scope defines the range of optimum performance of the animal, with maximum availability of aerobic energy to all physiological functions including growth and reproduction. Within the pejus

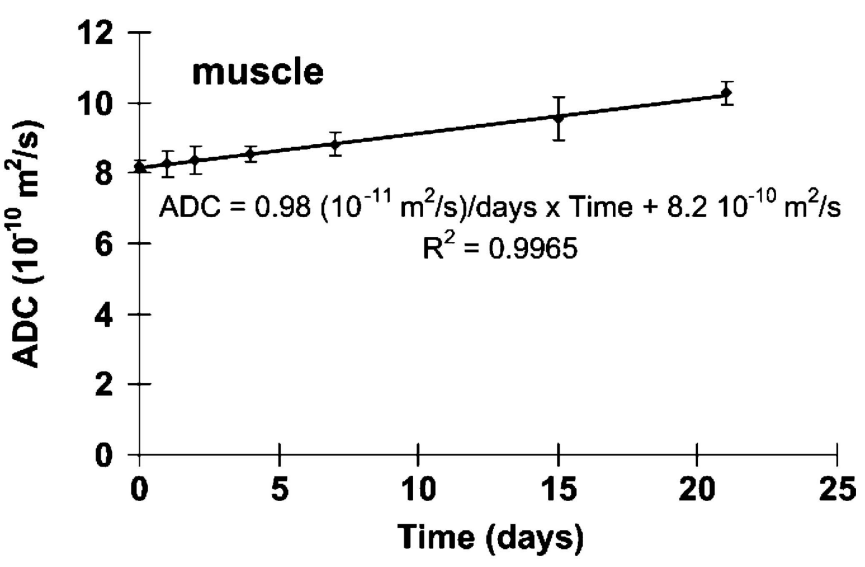

(getting worse) range, survival of the individual animal is still possible, but scopes for activity, growth and reproduction might already be diminished. In the critical temperature range, which is indicated by minimized aerobic scope and the onset of anaerobic metabolism, survival is time limited [2,33,34].

This hypothesis was tested using in vivo MR imaging and spectroscopy on a Bruker Biospec $47 / 40$ with a 40-cm magnet bore operating at $4.7 \mathrm{~T}$ in the stenothermal Antarctic eelpout, Pachycara brachycephalum [9]. The fish had an average length of $25 \mathrm{~cm}$, a mean weight of $50 \mathrm{~g}$ and were kept at $0 \pm 0.5^{\circ} \mathrm{C}$ and a salinity of $32.5 \%$. During the experiment water temperature was increased from $0^{\circ} \mathrm{C}$ (control) to $15^{\circ} \mathrm{C}$ by $1^{\circ} \mathrm{C} / 12 \mathrm{~h}$, whilst the fish remained in the magnet. To test the impact of oxygen limitations two experimental settings were tested, one normoxic $\left(P_{\mathrm{O}_{2}}: 20.3-21.3 \mathrm{kPa}\right)$ and one hyperoxic $\left(P_{\mathrm{O}_{2}}: 45 \mathrm{kPa}\right)$. Flow-weighted MR imaging (TOF) was applied to examine blood flow in the aorta dorsalis using the following parameters: matrix, $128 \times 128$; field of view, $4 \times 4 \mathrm{~cm} ; 5$ slices at $2 \mathrm{~mm}$ each; sweep width: $50 \mathrm{kHz}$; flip angle, $45^{\circ}$ $60^{\circ}$ (using a hermite pulse of $2000 \mu \mathrm{s}$ ); TR, $100 \mathrm{~ms}$; echo time (TE), $10 \mathrm{~ms}$; acquisition time, $1 \mathrm{~min} ; 2$ averages. In the obtained images, blood vessels were picked manually and changes in the ratio of signal intensity over noise 


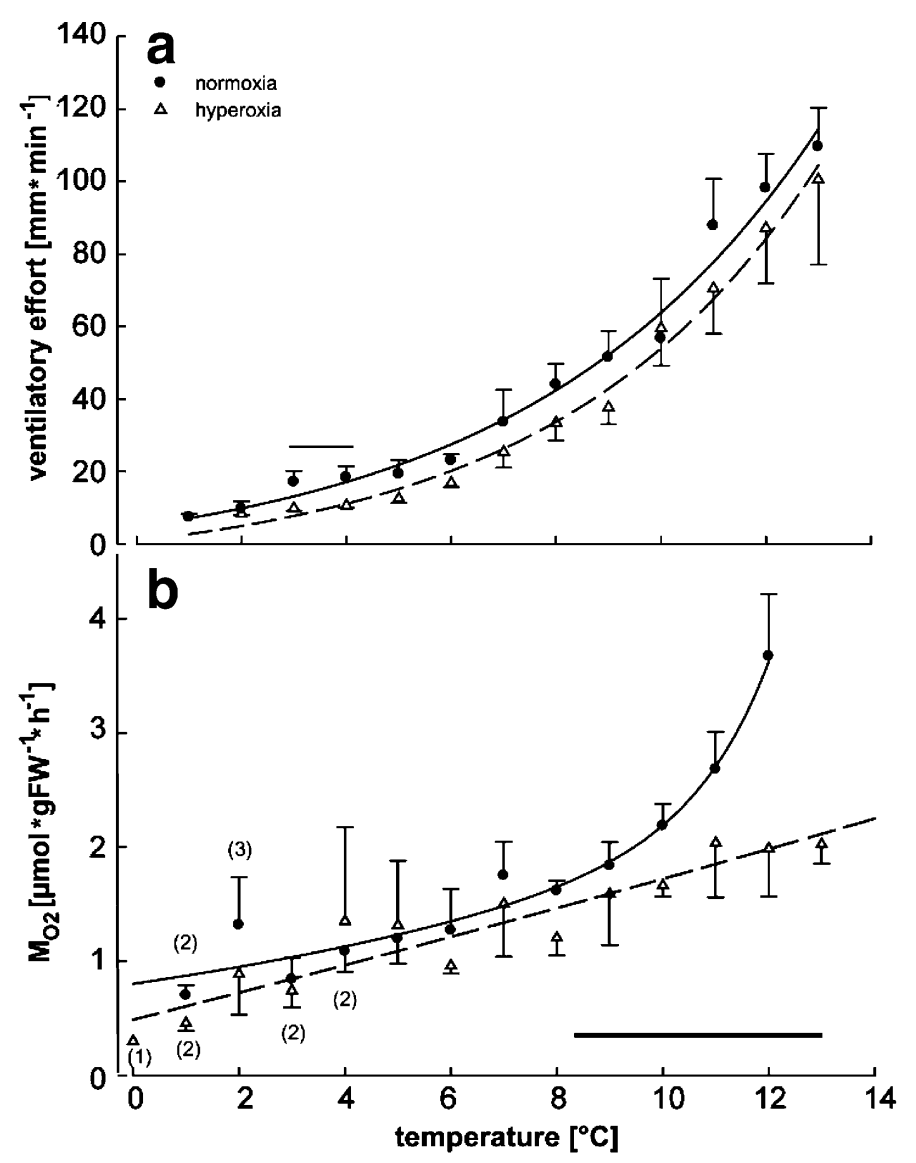

Fig. 7 a Ventilatory effort, b oxygen consumption $\left(\mathrm{M}_{02}\right)$, $\mathbf{c}$ arterial blood flow changes read from the aorta dorsalis and $\mathbf{d}$ white muscle $\mathrm{T}_{2}^{*}$ contrast (reflecting tissue oxygenation) acquired at different temperatures. Hyperoxic conditions (triangles) are compared with normoxic data (black circles). The horizontal bar parallel to the $x$-axis indicates significant differences between both series. The number of animals used per data point $(n)$ was (3-6) unless indicated otherwise in the figure. Asterisks represent significant differences above that temperature. Exponential and linear best fits are presented for $8 \mathrm{a}$ and $\mathrm{b}$ respectively. Line fits in $8 \mathrm{c}$ and $\mathrm{d}$ indicate an overall trend within the data sets (adapted from [9])

intensity were used to determine relative changes in blood flow. $\mathrm{T}_{2}^{*}$-weighted MR imaging estimated tissue oxygenation changes (BOLD contrast). Imaging parameters for BOLD MRI were as follows: matrix, $128 \times 128$; field of view, $4 \times 4 \mathrm{~cm}$; 5 slices at $2 \mathrm{~mm}$ each; sweep width, $50 \mathrm{kHz}$; flip angle, $11^{\circ}$ (pulse shape, sinc3; pulse length, $2000 \mu \mathrm{s}$ ); TR, $100 \mathrm{~s} ; \mathrm{TE}, 40 \mathrm{~ms}$; acquisition time, $4 \mathrm{~min} ; 4$ repetitions; 2 averages [9].

In vivo ${ }^{31} \mathrm{P}-\mathrm{NMR}$ spectroscopy was used for investigations of energy metabolism and acid-base regulation. In addition, classic oxygen consumption measurements and analyses of ventilatory effort were performed. Figure $3 \mathrm{~b}$ presents examples of typical transversal flow-weighted and $\mathrm{T}_{2}^{*}$-weighted MR images relative to a coronal anatomical MR image of the Antarctic eelpout. Different organs

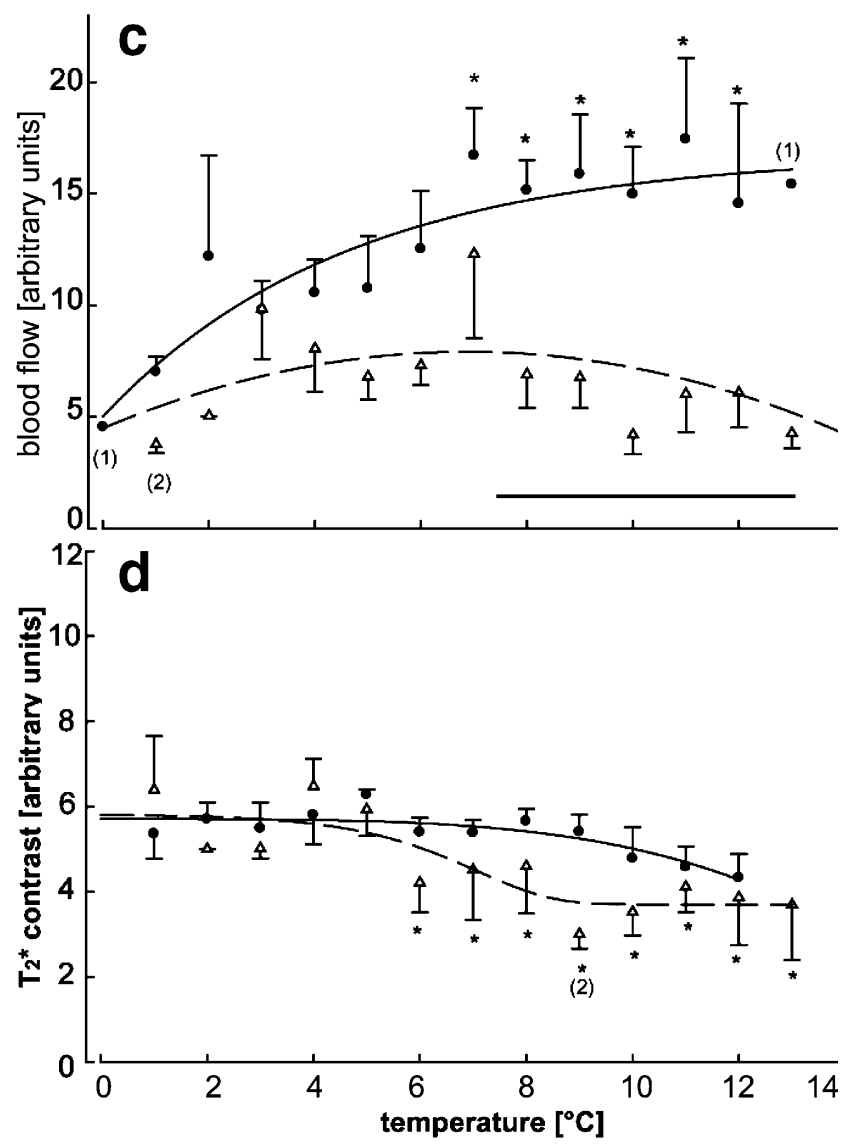

can be identified in both anatomical and $\mathrm{T}_{2}^{*}$-weighted images. In the flow-weighted image blood, vessels like the aorta dorsalis and the vena cava posterior are visible as bright spots. Figure 7 summarizes the results of ventilation and oxygen consumption measurements in comparison to blood flow analyses of the aorta dorsalis derived from flow-weighted MRI. Under normoxic conditions ventilatory effort and oxygen consumption increased exponentially with temperature. Blood flow also increased but levelled off above $7^{\circ} \mathrm{C}$. Tissue oxygenation in white muscle derived from $\mathrm{T}_{2}^{*}$-weighted $\mathrm{MR}$ imaging did not change significantly, despite increased oxygen demand during warming.

Under hyperoxic conditions ventilation still increased exponentially upon warming. Most importantly, the rise in oxygen consumption was reduced from an exponential slope under normoxia to a linear slope under hyperoxia. Blood flow did no longer change over the whole temperature range. Hyperoxia conditions revealed a significant decrease in oxygenation of white muscle tissue above $5--6^{\circ} \mathrm{C}$, which reflected the lower levels of blood flow and the lower whole animal $\mathrm{O}_{2}$ demand. The results indicate that, as a trade-off at higher temperatures visible under hyperoxia, blood perfusion to muscular tissue may be reduced so that oxygen can be delivered to more relevant aerobic organs such as the liver. Overall, the 
comparison of normoxic and hyperoxic conditions indicates that a temperature-induced rise in metabolic oxygen requirements was not adequately balanced by oxygen delivery through the cardiovascular system above $7^{\circ} \mathrm{C}$ under normoxia, resulting in a mismatch in oxygen delivery and demand. This imbalance leads to a loss in aerobic scope (see above). Elevated oxygen availability reduces temperature-dependent cardiovascular costs and thereby oxygen consumption, such that thermal stress is reduced. Altogether, this data set supports the principle of an oxygen-limited thermal tolerance in animals [2] and provides an excellent example for the application of in vivo MRI methods to study environmental physiology.

\section{MRI of teleost fish provides new insight into MRI contrast mechanisms}

Temperature dependence of brain apparent diffusion coefficient values

Most brain ADC data available in the literature have been obtained from mammals, and thus at $37^{\circ} \mathrm{C}$. Studying the impact of hypothermia on the ADC of rat brain, Jiang et al, [35] found that tissue ADC decreases at $1.5 \% /{ }^{\circ} \mathrm{C}$. Because of the narrow range of body temperatures usually displayed by mammals, fish represent a nice alternative to test the temperature dependence of tissue ADC values. This is illustrated in one study of carp [8], where ADC determined in carp brain at $18^{\circ} \mathrm{C}$ yielded values of $399 \pm 74 \times 10^{-12} \mathrm{~m}^{2} / \mathrm{s}$ for the common carp and $487 \pm 60 \times 10^{-12} \mathrm{~m}^{2} / \mathrm{s}$ for the crucian carp. Since temperature during the fish measurements was $19^{\circ} \mathrm{C}$ lower than mammalian body temperature, according to Jiang et al. [35], the resulting ADC values should be $28.5 \%$ lower $\left(429-526 \times 10^{-12} \mathrm{~m}^{2} / \mathrm{s}\right)$ than those measured in e.g. rat brain $\left(600-700 \times 10^{-12} \mathrm{~m}^{2} / \mathrm{s}\right)$. Using fish would allow one to obtain experimental ADC data from a wide range of temperatures and to compare them with the diffusion values of free water for a better understanding of what $\mathrm{ADC}$ values represent.

In vivo in situ assessment of the contribution of deoxyhaemoglobin concentration to the BOLD signal

BOLD measurements in tissue are complicated to interpret since alterations in cerebral blood flow $(\mathrm{CBF})$, cerebral blood volume (CBV) and the ratio of oxy- to deoxyhemoglobin all seem to play a role. The anoxiaresistant crucian carp is an excellent model to study the correlation between BOLD contrast in vivo in situ in the brain, and the $P_{\mathrm{O}_{2}}$ and deoxyhemoglobin concentration in the blood without any contaminating $\mathrm{CBV}$ and $\mathrm{CBF}$ changes within a broad range of $P_{\mathrm{O}_{2}}$ values. For a rough estimate it is sufficient to change the $P_{\mathrm{O}_{2}}$ of the environmental water between zero and normal to create a range of

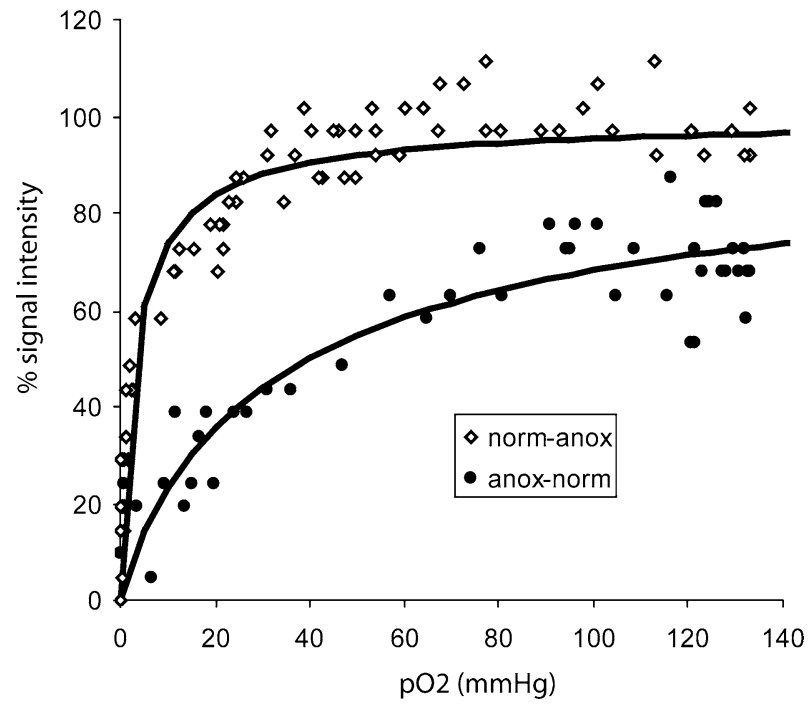

Fig. 8 Oxygen dissociation curves obtained in vivo and non-invasively at the level of the brain of a crucian carp. The fish was progressively transferred from normoxic to anoxic water (norm-anox) and vice versa (anox-norm) with a 1-h anoxic period in between. The entire procedure took about $3 \mathrm{~h}$, during which continuously GE images of the head of the carp were acquired providing BOLD SI changes $\left(y\right.$-axis) while the $P_{O 2}$ changes in the water $(x$-axis) were monitored online. A linear correlation was demonstrated between the $P_{O 2}$ in the water $(0$ - anoxia to $400 \mathrm{mmHg}$ - hyperoxia) and the blood of the fish (0 to $40 \mathrm{mmHg})\left(r^{2}=0.79\right)$ [38]

increasing $P_{\mathrm{O}_{2}}$ values in the blood leaving the gills. Since fish have a single-loop cardiovascular system, this blood will then go straight to the brain. Crucian carp is known to maintain $\mathrm{CBV}$ values during hypoxic challenge and to change its $\mathrm{CBF}$ only at blood oxygen concentrations lower than $0.1 \mathrm{mg} / \mathrm{l}$ oxygen in order to increase glucose supply to the brain [36]. Increasing CBF during anoxia will not affect BOLD contrast. Therefore crucian carp is an excellent model to study in vivo in situ oxygen dissociation curves using GE MRI sequences (TE/TR $=12 / 300 \mathrm{~ms}$, acquisition matrix of $128 \times 128$, FOV of $40 \mathrm{~mm}$, spectral width of $25 \mathrm{~Hz}, 1$ average, 7-T SMIS MRI instrument (UK)) during progressive water $P_{\mathrm{O}_{2}}$ alteration (Fig. 8). To investigate the $\mathrm{pH}$ sensitivity of the $\mathrm{O}_{2}$-hemoglobin equilibrium (Bohr effect) we challenged the fish within $1 \mathrm{~h}$ of anoxia, which is known to lower tissue $\mathrm{pH}$ from 7.8 to 7.4 [37] and observed a clear Bohr effect (Fig. 8) [38]. MRI in crucian carp provide data that MRI in mammals can never achieve due to the limited hypoxia tolerance of the mammalian brain.

\section{Explaining $\mathrm{T}_{2}$ and $\mathrm{ADC}$ changes using fish as a model}

Mammals do not sustain the major osmotic changes that would lead to substantial tissue ADC and $\mathrm{T}_{2}$ changes. Combined $T_{2}$ and ADC changes are therefore almost 
exclusively observed and reported from pathologies associated with stroke and other edema. Carp were submitted to an osmotic challenge resulting in massive ADC and $\mathrm{T}_{2}$ changes [7]. The MRI findings and the correlation with plasma osmolarity values obtained from the same fish illustrate how using fish as an animal model for MRI studies provides a new angle from which to understand MRI contrast changes.

\section{Future perspectives}

MRI of teleost fish provides new insights in MRI contrast mechanisms and the underlying physiological phenomena. In particular functional BOLD MRI in fish, which investigates brain-activity-induced BOLD contrast changes, would be interesting for several reasons. It is not yet known whether brain activity in ectothermic animals provokes a haemodynamic response in the first place and it is likely that the entire BOLD response might by slowed down due to the lower body temperature, allowing a better temporal resolution of the entire BOLD response. Even the temperature dependence of the haemodynamic response could be investigated in fish. From an evolutionary point of view it would be interesting to study the haemodynamic brain-activity-induced response by performing comparative functional MRI (fMRI) experiments in mammals, birds, amphibians and fish.

Functional MRI in fish brain in general would allow several interesting applications in which the fish brain model would then be used owing to its less complicated organization. This would definitely be relevant for those circuits whose mechanisms and molecules have been conserved throughout evolution. In this context it would be relevant to perform functional MRI at the level of the hypothalamic-pituitary-adrenal (HPA or stress) axis and the brain-pituitary-gonadal (BPG) axis using appropriate external stimuli such as alarm substances and sex attractants (both collected from fish), which can be administered in different doses to the external water.

Long-term anaesthesia [8] and long-term restraining of fish without anesthesia (up to $9 \mathrm{~h} \mathrm{-} \mathrm{[39])} \mathrm{is} \mathrm{feasible.}$ Even conscious fish without restraint can be maintained in the magnet for up to a week [9]. This opens the potential for in vivo MRI investigations which require either a long measuring time, such as diffusion tensor imaging, or the follow-up of slow changes.

From a more general point of view, examples illustrated in this review demonstrate that MRI can be extended to organs other than brain and also to an integrative study of organismic functioning. Such integrative studies will become more relevant in an effort to address the whole organism capacity of coping with environmental change.

In the end, this review should convince the reader that MRI of small teleost fish represents a valuable tool for integrative studies of organismic functioning and also provides the bonus of approaching MRI contrast mechanisms from a different angle.

\section{References}

1. Willmer P, Stone G, Johnston IA (2000) Environmental physiology of animals. Blackwell p 644

2. Pörtner HO (2001) Climate change and temperature dependent biogeography: oxygen limitation of thermal tolerance in animals. Naturwissenschaften 88:137-146

3. Pörtner HO (2002) Climate change and temperature dependent biogeography: systemic to molecular hierarchies of thermal tolerance in animals. Comp Biochem Physiol 132A:739-761

4. Pörtner HO (2004) Climate variability and the energetic pathways of evolution: the origin of endothermy in mammals and birds. Physiol Biochem Zool 77(6) (in press)

5. Hochachka PW, Somero GN (2002) Biochemical adaptation. Mechanism and process in physiological evolution. Oxford University Press, Oxford

6. Blackband SJ, Stoskopf MK (1990) In vivo nuclear magnetic resonance imaging and spectroscopy of aquatic organisms. MRI 8:191-198
7. Van der Linden A, Vanaudenhove M, Verhoye M, De Boeck G, Blust R (1999) Osmoregulation of common carp (cyprinus carpio) when exposed to an osmotic challenge assessed in-vivo and non-invasively by diffusion- and T2-weighted magnetic resonance imaging. CBP A124:343-352

8. Van der Linden A, Verhoye M, Nilsson GE (2001) Does anoxia induce cell swelling in carp brains? in vivo MRI measurements in crucian carp and common carp. J Neurophysiol 85:125-133

9. Mark FC, Bock C, Pörtner HO (2002) Oxygen limited thermal tolerance in Antarctic fish investigated by magnetic resonance imaging (MRI) and spectroscopy ( $\left.{ }^{31} \mathrm{P}-\mathrm{MRS}\right)$. Am J Physiol Regul Integr Comp Physiol 283:R1254-R1262

10. Bock C, Sartoris FJ, Pörtner HO (2002) In vivo MR spectroscopy and MR imaging on non-anaesthetized marine fish: techniques and first results. Magn Reson Imaging 20:165-172
11. Neustadter DM, Drushel RF, Chiel HJ (2002) Kinematics of the buccal mass during swallowing based on magnetic resonance imaging in intact, behaving Aplysia californica. J Exp Biol 205:939-958

12. Brouwer M, Engel DW, Bonaventura J, Johnson GA (1992) In vivo magnetic resonance imaging of the blue crab, callinectes sapidus: effect of cadmium accumulation in tissues on proton relaxation properties. J Exp Zool 263:32-40

13. Bock C, Frederich M, Wittig RM, Pörtner HO (2001) Simultaneous observation of haemolymph flow and ventilation in marine spider crabs at different temperatures: a flow weighted MRI study. Magn Reson Imaging 19:1113-1124

14. Fernández M, Bock C, Pörtner HO (2000) The cost of being a caring mother: the ignored factor in the reproduction of marine invertebrates. Ecol Lett 3:487-494 
15. Van den Thillart G, Van Waarde A, Muller HJ, Erkelens C, Lugtenburg J (1989) A flow through probe for in vivo

${ }^{31} \mathrm{P}$ NMR Spectroscopy of unanesthetized aquatic vertebrates at 9.4 Tesla. J Magn Reson 84:573-579

16. Kugel $H$ (1988) Improving the signal-to-noise ratio of NMR signals by reduction of inductive losses. J Magn Reson 91:179-185

17. Bock C, Sartoris FJ, Wittig RM, Pörtner HO (2001) Temperature dependent $\mathrm{pH}$ regulation in stenothermal Antarctic and eurythermal temperate eelpout (Zoarcidae): an in vivo NMR study. Polar Biol 24:869-874

18. Sartoris FJ, Bock C, Pörtner HO (2003) Temperature dependent $\mathrm{pH}$ regulation in eurythermal and stenothermal marine fish: an interspecies comparison using ${ }^{31}$ P-NMR. J Therm Biol 28:363-371

19. Pörtner HO, Mark FC, Bock C (2004) Oxygen limited thermal tolerance in fish? Answers obtained by nuclear magnetic resonance techniques. Respir Physiol Neurobiol 141:243-260

20. Lutz PL, Nilsson GE (1997) Contrasting strategies for anoxic brain survival-glycolysis up or down. J Exp Biol 200:411-419

21. Van den Thillart G, Van Waarde A (1991) $\mathrm{pH}$ changes in fish during environmental anoxia and recovery: the advantages of the ethanol pathway. In: Woakes AJ, Grieshaber M, Bridges CR (eds) Physiological strategies for gas exchange and metabolism. SEB Seminar Series: 173-190

22. Van Ginneken V, Nieveen M, Van Eersel R, Van den Thillart G, Addink A (1996) Neurotransmitter levels and energy status in brain of fish species with and without the survival strategy of metabolic depression. Comp Biochem Physiol A 114:189-196

23. Benveniste H, Hedlund LW, Johnson GA (1992) Mechanism of detection of acute cerebral ischemia in rats by diffusion weighted magnetic resonance microscopy. Stroke 23(5):746-754

24. Mintorovitch J, Moseley ME, Chileuitt L, Shimizu H, Cohen Y, Weinstein PR (1991) Comparison of diffusion- and $\mathrm{T}_{2}$-weighted MRI for the early detection of cerebral ischemia and reperfusion in rats. Magn Reson Med 18:39-50
25. Moseley ME, Cohen Y, Mintorovitch J (1990) Early detection of regional cerebral ischemia in cats: comparison of diffusion-and $\mathrm{T}_{2}$-weighted $\mathrm{MRI}$ and spectroscopy. Magn Reson Med 14:330-346

26. Ebisu T, Naruse S, Horikawa Y, Ueda $\mathrm{S}$, Tanake C, Uto M, Umeda M, Higuchi T (1993) Discrimination between different types of white matter oedema with diffusion-weighted MR imaging. J Magn Reson Imaging 3:863-868

27. Sevick RJ, Kanda F, Mintorovitch J, Arieff AI, Kucharczyk J, Tsuruda JS, Norman D, Mosely ME (1992) Cytotoxic brain oedema: assessment with diffusion-weighted MR imaging. Radiology 185:687-690

28. Assem H, Hanke W (1979) Volume regulation of muscle cells in the euryhaline teleost, Tilapia mossambica. Comp Biochem Physiol 64A:17-23

29. Schmidt-Nielsen B (1975) Comparative physiology of cellular ion and volume regulation. J Exp Zool 194:207-220

30. Schmidt-Nielsen B (1977) Volume regulation of muscle fibres in the killifish, Fundulud heteroclitus. J Exp Zool 199:411-418

31. Gupta OP, Hanke W (1982) The effects of osmotic stressors on the stenohaline carp (Cyprinus carpio). Comp Biochem Physiol 71A:165-173

32. Pörtner HO (2002) Physiological basis of temperature-dependent biogeography: trade-offs in muscle design and performance in polar ectotherms. J Exp Biol 205:2217-2230

33. Pörtner HO, Hardewig I, Sartoris FJ, van Dijk PLM (1998) Energetic aspects of cold adaptation: critical temperatures in metabolic, ionic and acid-base regulation? In: Pörtner HO, Playle R (eds) Cold ocean physiology. Cambridge University Press, Cambridge, pp 88-120
34. Frederich M, Pörtner HO (2000) Oxygen limitation of thermal tolerance defined by cardiac and ventilatory performance in spider crab, Maja squinado. Am J Physiol; Regul Integr Comp Physiol 279:R1531-R1538

35. Jiang Q, Chopp M, Zhang ZG, Helpern JA, Ordidge RJ, Ewing J, Jiang P, Marchese B (1994) The effect of hypothermia on transient focal ischemia in rat brain evaluated by diffusion and perfusion weighted NMR imaging. J Cereb Blood Flow Metab 14:732-741

36. Nilsson GE, Hylland P, Lofman CO (1994) Anoxia and adenosine induce increased cerebral blood flow in crucian carp. Am J Physiol Regul Integr Comp Physiol 267: R590-R595

37. Van Waarde A, De Graaff I, Van den Thillart G, Erkelens C (1991) Acidosis (measured by nuclear magnetic resonance) and ethanol production in anoxic goldfish acclimated to $5^{\circ} \mathrm{C}$ and $20^{\circ}$ C. J Exp Biol 159:387-405

38. Van der Linden A, Verhoye $M$, Verschaeren I, Nilsson G, Berenbrink M (1999) Does BOLD Magnetic Resonance Imaging provides a tool to measure oxygen affinity of hemoglobin in vivo, in situ? A study in crucian carp. In: Proceedings of the European Society for Comparative Physiology and Biochemistry Conference, Arhus, pp 27-30

39. Borger R, De Boeck G, Van Audekerke J, Dommisse R, Blust R, Van der Linden A (1998) Recovery of the energy metabolism after a hypoxic challenge at different temperature conditions: a ${ }^{31} \mathrm{P}$ nuclear magnetic resonance spectroscopy study with common carp. CBP A 120:143-150 